\title{
Covid-19 and ageing: four alternative conceptual frameworks
}

\author{
Davide Serpico $^{1}$ (D) M. Cristina Amoretti ${ }^{1}$
}

Received: 29 December 2020 / Accepted: 14 June 2021 / Published online: 29 June 2021

(C) Springer Nature Switzerland AG 2021

\begin{abstract}
Ageing is one of the main risk factors for Covid-19. In this paper, we delineate four alternative conceptualisations of ageing, each of which determines different understandings of its causal role to the susceptibility to Covid-19 as well as to the severity of its symptoms and adverse health outcomes.
\end{abstract}

Keywords Covid-19 $\cdot$ Ageing $\cdot$ Risk factor

Ageing has been identified as one of the main risk factors for Covid-19, severe forms of the disease, and adverse health outcomes (Chen et al., 2021; Ho et al., 2020). However, taking ageing as a risk factor of Covid-19 leaves it open the question of whether the association between Covid-19 and ageing reflects any causal relationship: does ageing causes the higher susceptibility to Covid-19 or higher symptoms' severity? Alternatively, is the association between ageing and severe forms of Covid-19 mediated by a third (or more) causal factor(s), as in the case of spurious correlations? As philosophers of science, we are interested in evaluating whether or not the relationship between Covid-19 and ageing may be conceived as causal. This is not only of theoretical but also of practical importance, as it can have major repercussions on how the elderlies are regarded during the current pandemic.

In what follows, we will contextualise the relationship between Covid-19 and ageing within four views of the ontological status of ageing that reflect recent trends in the metaphysics of biomedical sciences: first, ageing as a pathogenic mechanism;

Davide Serpico

davide.serpico@unige.it

M. Cristina Amoretti

cristina.amoretti@unige.it

1 Department of Classics, Philosophy and History, University of Genoa, Via Balbi, 4, 16126 Genoa, Italy 
second, as a Homeostatic Property Cluster (HPC); third, as a Stable Property Cluster (SPC); fourth, as a causal network. Notably, these four views involve different understandings of the connection between Covid-19, the various hallmarks that are typically associated with ageing, and the causal mechanisms relating to such features; they thus generate different causal explanations of why ageing is statistically associated with severe forms of Covid-19. Specifically, we will defend the interpretation of ageing as a causal network, according to which the association between ageing and severe forms of Covid-19 is due to a network of immunological and physiological properties that realises ageing and indirectly affects the susceptibility to severe forms of Covid-19. Not only this interpretation accommodates recent empirical findings, but as it implies that ageing and Covid-19 both are effects of some other cause(s), it also brings positive upshots on the ethical-social side.

Let us start with the hypothesis that ageing itself is a direct cause of severe forms of Covid-19. This view can be supported by an ontological framework that identifies ageing with a general biological mechanism that causes the various features of ageing. For instance, such mechanism could be the progressive decline of all biological functions or the loss of physiological integrity. If so, statements about ageing as a risk factor of Covid-19 can be read causally, in terms of how this general mechanism increases the susceptibility to Covid-19 or contributes to the symptoms' higher severity and adverse health outcomes. This reductive framework seems to us the only one that would make sense of statements about ageing as the cause (or one of the causes) of severe forms of Covid-19: as we will discuss shortly, no other conceptualisation of ageing would allow one to say that ageing per se has any causal role in Covid-19.

An alternative view takes ageing as causally connected to Covid-19, though indirectly. Here, ageing can be seen as a cluster of interconnected properties, such as genomic instability, epigenetic alterations, cellular senescence (Lopez-Otin et al., 2013), and the dysregulation of immunological functions. If so, ageing cannot be reduced to any of such properties as it is the whole set of them. Two theories of clusters, which differ on the emphasis they put on causal processes and explanations, can support this view.

The first theory would take ageing as a cluster of properties that are causally interconnected thanks to some sort of causal process. This interpretation can be elucidated in terms of Boyd's HPC theory (1991), according to which properties in a property cluster are held together by a homeostatic mechanism-namely, a mechanism that takes together the properties that are typically associated to the cluster but allows slight variations in different instances of the cluster. Here, a given homeostatic mechanism, such as the progressive decline of some biological functions, would be what holds together the various properties characterising ageing but would also be the cause of other features to which ageing is statistically associated, including severe forms of Covid-19. In this case, ageing would have an indirect causative role: it would be associated with severe forms of Covid-19 because the homeostatic mechanism that holds together the properties characterising ageing has some causal effect on the severity of Covid-19 symptoms.

The second theory would conceptualise ageing as a Stable Property Cluster (Slater, 2015), meaning that ageing is a cluster of properties that tend to be 
statistically associated with each other. The main difference with the HPC view is that the SPC account would advise not to seek a causal explanation of how a specific mechanism holds together the properties of ageing (Onishi \& Serpico, 2021). Thus, this view would have limited explanatory potential on why ageing is associated with severe forms of Covid-19, as for the classical notion of risk factor.

Finally, Covid-19 and ageing can be regarded as parts of the same causal network, namely, a network of immunological, physiological, and psychological parameters that realises both ageing and severe forms of Covid-19. An ontological framework for this view can be provided by Khalidi's Causal Network Node theory (Khalidi, 2018). Here, ageing could not be reduced to any single node of the network, nor to any specific causal mechanism; thus, the question of the causal role of ageing in Covid-19 would concern the relationship between Covid-19 and the nodes of the network that realises ageing, rather than ageing per se. In other words, ageing would be regarded as an effect of a causal network that involves both ageing and Covid-19, rather than the cause of the greater susceptibility to Covid-19 and severe forms of the disease. If so, the actual causes would be (some of) the elements of the causal network themselves - which nodes is a question that is to be empirically addressed.

We believe that the latter ontological framework can better account for the available data about the many risk factors of Covid-19. For instance, it nicely fits with recent results from physiology research, which has identified higher levels of Covid19 symptoms severity in individuals with higher inflammatory parameters typical of ageing, but also of other pathologies associated with ageing, such as diabetes, obesity, and major depression (Cunha et al., 2020; Hazeldine \& Lord, 2020; Meftahi et al., 2020). Such research refers to the concepts of immunosenescence and "inflamm-aging," which denote the dysregulation of many immune functions during ageing that can lead to the cytokine release syndrome (a phenomenon also known as "cytokine storm") as well as chronic systemic inflammation that contributes to the increased susceptibility of the elderlies to infections.

Interestingly, the concepts of immunosenescence and inflamm-aging bring attention to the temporality of physiological development, as both such physiological phenomena tend to occur over time and, for precisely this reason, are associated with ageing. This would explain why ageing is associated with severe forms of Covid-19 without implying any causative role of ageing, neither direct nor indirect: indeed, the causal network connected to Covid-19 would not include ageing itself, but rather unbalanced chronic inflammation that is also part of the network that realises ageing.

Further investigation on the ontological status of ageing will help clarify the relationship between Covid-19, ageing, and other risk factors. We believe that understanding Covid-19 and ageing as parts of the same causal network can not only enhance our understanding of the aetiology of Covid-19, but also have important ethical and social consequences: for instance, conceptualising ageing as an effect, rather than a cause, can help prevent forms of discrimination and stigmatisation against the elderlies related to the allegedly harmful role of ageing in the current pandemic. 
Funding This research was supported by the Department of Classics, Philosophy and History of the University of Genoa.

\section{Declarations}

Conflict of interest The authors declare that they have no conflict of interest.

\section{References}

Boyd, R. (1991). Realism, anti-foundationalism and the enthusiasm for natural kinds. Philosophical Studies, 61, 127-148.

Chen, Y., Klein, S. L., Garibaldi, B. T., Li, H., Wu, C., Osevala, N. M., Li, T., Margolick, J. B., Pawelec, G., \& Leng, S. X. (2021). Aging in COVID-19: Vulnerability, immunity and intervention. Ageing Research Reviews, 65, 101205.

Cunha, L. L., Perazzio, S. F., Azzi, J., Cravedi, P., \& Riella, L. V. (2020). Remodeling of the immune response with aging: Immunosenescence and its potential impact on COVID-19 immune response. Frontiers in Immunology. https://doi.org/10.3389/fimmu.2020.01748

Hazeldine, J., \& Lord, J. M. (2020). Immunesenescence: A predisposing risk factor for the development of COVID-19? Frontiers in Immunology, 11, 2381.

Ho, F. K., Petermann-Rocha, F., Gray, S. R., Jani, B. D., Katikireddi, S. V., Niedzwiedz, C. L., Foster, H., Hastie, C. E., Mackay, D. F., Gill, J. M. R., O’Donnell, C., Welsh, P., Mair, F., Sattar, N., CelisMorales, C. A., \& Pell, J. P. (2020). Is older age associated with COVID-19 mortality in the absence of other risk factors? General population cohort study of 470,034 participants. PloS One, 15(11), e0241824.

Khalidi, M. A. (2018). Natural kinds as nodes in causal networks. Synthese, 195(4), 1379-1396.

López-Otín, C., Blasco, M. A., Partridge, L., Serrano, M., \& Kroemer, G. (2013). The hallmarks of aging. Cell, 153(6), 1194-1217.

Meftahi, G. H., Jangravi, Z., Sahraei, H., \& Bahari, Z. (2020). The possible pathophysiology mechanism of cytokine storm in elderly adults with COVID-19 infection: The contribution of "inflame-aging." Inflammation Research, 69, 825-839.

Onishi, Y., \& Serpico, D. (2021). Homeostatic property cluster theory without homeostatic mechanisms: Two recent attempts and their costs. Journal for General Philosophy of Science, 1-22.

Slater, M. H. (2015). Natural kindness. The British Journal for the Philosophy of Science, 66(2), $375-411$.

Publisher's Note Springer Nature remains neutral with regard to jurisdictional claims in published maps and institutional affiliations. 